\title{
Factor XII in PMM2-CDG patients: role of N-glycosylation in the secretion and function of the first element of the contact pathway
}

\author{
Raquel López-Gálvez', María Eugenia de la Morena-Barrio ${ }^{1 *}$ (D), Alberto López-Lera ${ }^{2,3}$, Monika Pathak \\ Antonia Miñano ${ }^{1}$, Mercedes Serrano ${ }^{5}$, Delphine Borgel ${ }^{6,7}$, Vanessa Roldán ${ }^{1}$, Vicente Vicente ${ }^{1}$, Jonas Emsley ${ }^{4}$ \\ and Javier Corral ${ }^{1}$
}

\begin{abstract}
Background: Congenital disorders of glycosylation (CDG) are rare diseases with impaired glycosylation and multiorgan disfunction, including hemostatic and inflammatory disorders. Factor XII (FXII), the first element of the contact phase, has an emerging role in hemostasia and inflammation. FXII deficiency protects against thrombosis and the p.Thr309Lys variant is involved in hereditary angioedema through the hyperreactivity caused by the associated defective O-glycosylation. We studied FXII in CDG aiming to supply further information of the glycosylation of this molecule, and its functional and clinical effects. Plasma FXII from 46 PMM2-CDG patients was evaluated by coagulometric and by Western Blot in basal conditions, treated with N-glycosydase F or activated by silica or dextran sulfate. A recombinant FXII expression model was used to validate the secretion and glycosylation of wild-type and variants targeting the two described FXII N-glycosylation sites (p.Asn230Lys; p.Asn414Lys) as well as the p.Thr309Lys variant.

Results: PMM2-CDG patients had normal FXII levels (117\%) but high proportions of a form lacking N-glycosylation at Asn414. Recombinant FXII p.Asn230Lys, and p.Asn230Lys\&p.Asn414Lys had impaired secretion and increased intracellular retention compared to wild-type, p.Thr309Lys and p.Asn414Lys variants. The hypoglycosylated form of PMM2CDG activated similarly than FXII fully glycosylated. Accordingly, no PMM2-CDG had angioedema. FXII levels did not associate to vascular events, but hypoglycosylated FXII, like hypoglycosylated transferrin, antithrombin and FXI levels did it.

Conclusions: N-glycosylation at Asn230 is essential for FXII secretion. PMM2-CDG have high levels of FXII lacking N-glycosylation at Asn414, but this glycoform displays similar activation than fully glycosylated, explaining the absence of angioedema in CDG.
\end{abstract}

Keywords: Factor XII, Congenital disorders of glycosylation, N-glycosylation, Angioedema, Hemostasis

\footnotetext{
*Correspondence: uge2985@hotmail.com

${ }^{1}$ Servicio de Hematología y Oncología Médica, Hospital Universitario Morales Meseguer, Centro Regional de Hemodonación, Universidad de Murcia, IMIB-Arrixaca, CIBERER, Ronda de Garay S/N, 30003 Murcia, Spain

Full list of author information is available at the end of the article
}

\section{Introduction}

There is a renaissance in studying the role of factor XII (FXII) in pathways leading to thrombosis and inflammation. No bleeding is associated with FXII deficiency observed in different animal models (mice and cats) [1, 2], and in FXII-deficient patients [3], which has dramatically changed by the observed antithrombotic protection caused by the severe reduction of FXII levels original author(s) and the source, provide a link to the Creative Commons licence, and indicate if changes were made. The images or other third party material in this article are included in the article's Creative Commons licence, unless indicated otherwise in a credit line to the material. If material is not included in the article's Creative Commons licence and your intended use is not permitted by statutory regulation or exceeds the permitted use, you will need to obtain permission directly from the copyright holder. To view a copy of this licence, visit http://creativecommons.org/licenses/by/4.0/. The Creative Commons Public Domain Dedication waiver (http://creativeco mmons.org/publicdomain/zero/1.0/) applies to the data made available in this article, unless otherwise stated in a credit line to the data. 
in mice [4-6], rats [7], rabbit [8, 9], or baboons [10]. Additionally, different FXII variants affecting the prolinerich domain, particularly the recurrent Thr309Lys mutation, have been recently involved in a type of lifethreatening inherited swelling disorder, hereditary angioedema (HAE) [11-14].

FXII is a precursor of a serine protease that initiates the procoagulant and proinflammatory protease cascades. It becomes autoactivated following binding to negatively charged either artificial or biological surfaces. Kallikrein is also able to convert FXII into an active protease. Activated FXII (FXIIa) may promote both the activation of its procoagulant substrate factor XI (FXI), and the release of the proinflammatory mediator bradikinin (BK) by kallikrein-mediated cleavage of high molecular weight kininogen [15].

Glycosylation is a common post-translational modification (PTM) that plays key roles in folding, secretion, stability and function of multiple glycoproteins [16]. This PTM may be particularly relevant for FXII. Actually, the impaired O-glycosylation caused by the p.Thr309Lys mutation has been involved in the increased sensibility of this variant to become activated [17].

Type 1 congenital disorders of glycosylation (CDG) is a wide and heterogeneous group of rare autosomal recessive disorders that have impaired the $\mathrm{N}$-glycosylation due to defects affecting one out of up to 25 different enzymes involved in the generation of the $\mathrm{N}$-glycan precursor [18]. The most common, although still very rare CDG, is PMM2-CDG, caused by mutations in $P M M 2$, which encodes the phosphomannomutase 2, an enzyme involved in the transformation of mannose6-phosphate into mannose-1-phosphate, one of the first steps of N-glycosylation [19]. Thus, PMM2-CDG patients have increased levels of hypoglycosylated forms of multiple $\mathrm{N}$-glycoproteins, particularly of hepatic origin. Actually, the identification of hypoglycosylated forms of transferrin is one gold standard in the diagnosis of PMM2-CDG [20].

We proposed that the analysis of FXII, a hepatic $80 \mathrm{kDa}$ glycoprotein containing $2 \mathrm{~N}$-glycosylation sites (one in the heavy chain -Asn230- and the other one in the light chain -Asn414- Fig. 1), in a large cohort of PMM2-CDG patients together with a recombinant model deleting the two N-glycosylation signals might help to understand the role of N-glycosylation in FXII, and the clinical impact of an impaired N-glycosylation of this molecule in PMM2CDG patients.

\section{Methods}

\section{Patients}

Forty-six patients with confirmed PMM2-CDG were retrospectively recruited from 6 hospitals [Spain (2),
France (2), Portugal (1), and Belgium (1)]. The clinical characteristics of the main cohort of these patients are described elsewhere [21]. Glycoforms of transferrin; antithrombin activity and FXI levels were quantified by HPLC, a chromogenic assay, and a coagulometric method, as described previously [22].

\section{Genetic analysis}

The PMM2-CDG variants were identified by NGS methods. Moreover, the rs1801020 polymorphism affecting the Kozak sequence of $F 12$ that significantly modulates the levels of FXII in plasma [23], was genotyped by a TaqMan assay (C__1989313_20) (ThermoFisher).

\section{Blood collection}

Blood samples were collected in citrate-anticoagulated tubes (1:10) and processed immediately. The tubes were centrifuged at $2500 \mathrm{rpm}$ for $20 \mathrm{~min}$ at room temperature and the separated plasma was aliquoted and stored at $-70{ }^{\circ} \mathrm{C}$ until analysis.

Genomic DNA was purified by the salting out following Puregen Blood Core Kit (Quiagen) procedure and stored at $-20^{\circ} \mathrm{C}$.

\section{Ethical issues}

The study was performed in accordance with the principles of the Declaration of Helsinki and approved by the ethics committee of Universitario Reina Sofía $(8 / 2013)$. Written informed consent from patients and healthy subjects was provided.

\section{FXII evaluation in plasma}

\section{Functional and antigenic evaluation of FXII}

The coagulant levels of FXII in plasma (FXII: C) of PMM2-CDG patients were measured using a coagulometric method in an automatic analyzer ACL TOP 700 after activating the contact pathway with micronized silica (SynthASil; 0020006800, HemosIL ${ }^{\circledR}$, Instrumentation Laboratory) and diluting the plasma of patients in a factor XII deficient plasma (0020011210, HemosIL ${ }^{\circledR}$, Instrumentation Laboratory). Results were shown as percentage of a reference plasma (Instrumentation Laboratory).

Antigen levels and forms of plasma FXII in PMM2CDG patients were studied by Western Blot using denaturing polyacrilamide electrophoresis (8\% PAGE) in the presence and absence of reducing agents (DTT). The blotting was performed by using a goat antihuman FXII polyclonal antibody (GAFXII-AP, Enzyme Research Laboratories), which only recognizes the heavy chain, followed by the goat anti-IgG secondary antibody coupled to peroxidase (A-9452-1VL, 
FXII WT

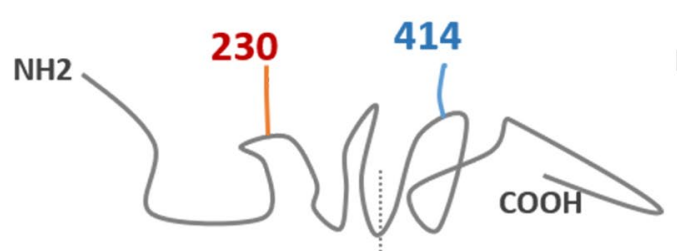

Heavy Chain $\%$ Light Chain

FXII -414

(p.Asn414)

NH2

230

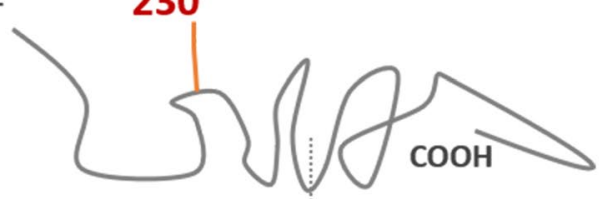

Heavy Chain $\quad \%$ Light Chain
FXII -230

(p.Asn230)

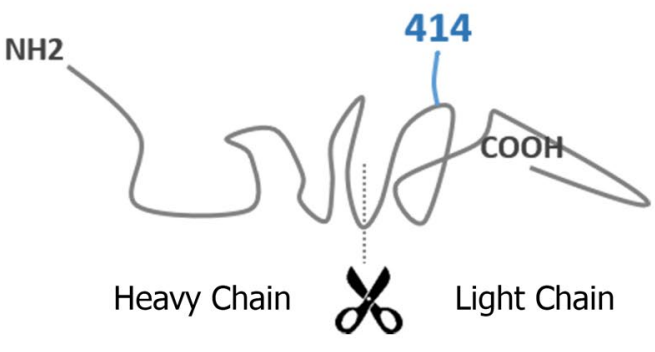

FXII -230/414

(p.Asn230 + p.Asn414)

$\mathrm{NH} 2$

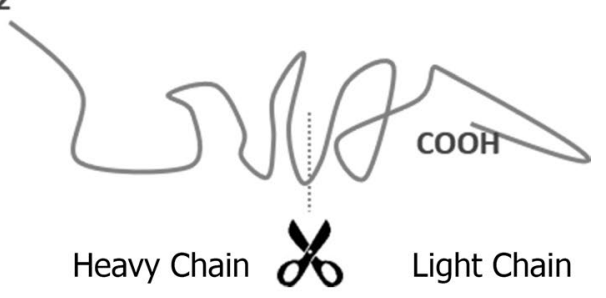

FXII 309

(p.Thr309Lys)

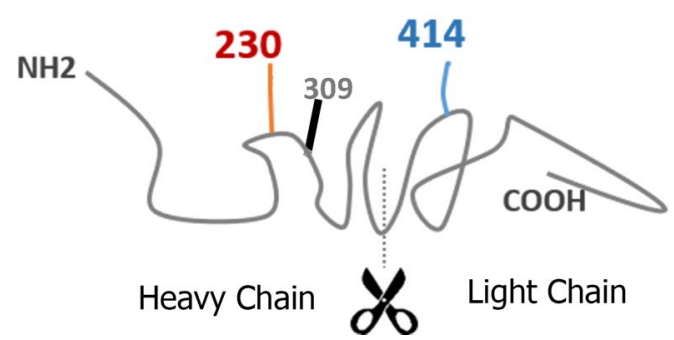

Fig. $1 \mathrm{~N}$-glycan sequons identified in FXII and FXII variants generated in the recombinant model. N-glycosylation positions are located in positions 230 (heavy chain) and 414 (light chain). The cleavage site of the heavy chain generated by activation of FXII is shown. As a control, the mutation involved in FXII-HAE p.Thr309Lys, also located in the heavy chain, was also generated

Sigma-Aldrich) as secondary antibody. Peroxidase was detected with a chemiluminescence system (ECL ${ }^{\mathrm{TM}}$, GE Healthcare) in an Image Quant LAS4000mini equipment (ExonBiotec, GE Healthcare).

Glycosylation of other hepatic proteins, antithrombin, $\alpha 1$-antitrypsin, FXI and transferrin was evaluated by Western Blot, Q-TOF, or HPLC as described elsewhere [22].

Densitometric analysis of glycoform bands was performed using Image J [24].

\section{Analysis of FXII N-glycosylation}

To characterize the N-glycan content of FXII, plasma of patients and controls $(2 \mu \mathrm{L})$ were denatured for $5 \mathrm{~min}$ at $100{ }^{\circ} \mathrm{C}$ in $150 \mathrm{mM}$ sodium phosphate buffer, pH 7.4 and 10\% NP-40. Subsequently, samples were treated with $2 \mathrm{U}$ of $\mathrm{N}$-glycosidase F (PNGase-F) (Roche Diagnostics $\mathrm{GmbH}$,) at $37{ }^{\circ} \mathrm{C}$ for $15 \mathrm{~h}$ and analyzed by Western Blotting as described before. 


\section{Activation of plasma FXII}

Two different agents were used to activate plasma FXII: i) micronized silica (SynthAsil 0,020,006,800, HemosIL ${ }^{\circledR}$, Instrumentation Laboratory) at different dilutions (stock solution and 1/10,1/20 and 1/50 dilutions), for $10 \mathrm{~min}$ at $37^{\circ} \mathrm{C}$; ii) dextran sulfate (DXS) (Pharmacia) from $0.2 \mu \mathrm{g} /$ $\mathrm{mL}$ to $0.001 \mu \mathrm{g} / \mathrm{mL}$, for $30 \mathrm{~min}$ at $37^{\circ} \mathrm{C}$. The activation of FXII was then evaluated by Western Blotting, as described before.

\section{Recombinant FXII}

We used the hFXII-full plasmid, which contains the cDNA sequence of human the $F 12$ gene. Four mutants were generated by site directed mutagenesis (Quik Change II Site-Directed Mutagenesis kit, Agilent technologies). The two N-glycosylation sites of FXII were abolished by changing the Asparagine (Asn) AAC codon, at position 230 or 414, to the Lysine (Lys) AAA codon. The two single and the double mutants were generated: p.Asn230Lys, p.Asn414Lys and p.Asn230Lys\&Asn414Lys. As an additional control, the p.Thr309Lys mutation was also generated (Fig. 1).

The oligonucleotides used for site directed mutagenesis are shown in Table 1. All the mutants generated were verified by Sanger sequencing.

Drosophila Schneider 2 (S2) cells were cultured in complete Drosophila Schneider medium supplemented with $10 \%$ fetal bovine serum at $28{ }^{\circ} \mathrm{C}$ (Gibco; ThermoFisher Scientific). Transfection of these cells with the appropriate plasmids $(30 \mathrm{ng} / \mu \mathrm{l})$ in HEPES buffered saline solution (50 mM HEPES, $1.5 \mathrm{mM} \mathrm{Na} 2 \mathrm{HPO} 4,280 \mathrm{mMNaCl}$, $\mathrm{pH}$ 7.1) was carried out with the calcium phosphate method following the manufacturer's protocol (Calcium Phosphate Transfection Kit; ThermoFisher Scientific). The cells were cultured for an additional $48 \mathrm{~h}$ before the addition of puromycin, which allows the selection of cells expressing the plasmid. The stable cells were then adapted to insect culture medium Serum-free Express Five (SFM) (Invitrogen). Subsequently, the SFM medium

\section{Table 1 Oligonucleotides used to carry out the site directed mutagenesis in the human F12 CDNA that remove the two N-glycosylation sites}

\begin{tabular}{ll}
\hline Mutation & Oligonucleotide sequence $\left(\mathbf{5}^{\prime} \mathbf{- \mathbf { 3 } ^ { \prime } )}\right.$ \\
\hline Asn230Lys & Forward: CACCTACCGGAAAGGACTGCCGAG \\
& Reverse: CTCGGCAGTCACTTTCCGGTAGGTG \\
Asn414Lys & Forward: CAGGAACGCCGTAAACACAGCTGTGAGC \\
& Reverse: GCTCACAGCTGTGTTACGGCGTTCCG \\
Thr309Lys & Forward: GAAGCCTCAGCCCAAGACCCGGACCCCGC \\
& Reverse: GCGGGGTCGGGTCTTGGGCTGAGGTTC \\
\hline
\end{tabular}

containing the secreted proteins was collected due to the induction of the cells with $\mathrm{CuSO}_{4}$, and the cell pellet was removed by centrifugation.

\section{Statistical analysis}

Statistical analysis were performed using the SPSS software (version 15.0). Correlation studies between the degree of hypoglycosylation and asialotransferrin or antithrombin levels were analysed using Pearson test. Mean comparisons were performed with T-Student test as variables showed normal distribution. A $p$ value $<0.05$ was considered statistically significant.

\section{Results}

FXII levels in CDG patients

PMM2-CDG patients showed a median FXII:C of 117\% [IQ range $86-152 \%$ ]. All patients presented FXII values within the normal range, except for 3 cases with lower levels (50-60\%). These 3 patients were homozygous TT for the functional rs 1801020 polymorphism.

The study of plasma FXII in our cohort of PMM2-CDG patients by Western Blot confirmed that FXII levels in patients were in the normal range and correlated with the $r s 1801020$ genotype, as carriers of the $\mathrm{C} / \mathrm{C}$ genotype had almost twice FXII in plasma than carriers of the T/T genotype (Data not shown). However, the most remarkable finding from these studies was the presence of a FXII form with faster electrophoretic mobility in SDS gels in all PMM2-CDG patients. This aberrant form was about $2 \mathrm{kDa}$ smaller than the wild-type according to the data observed in SDS-PAGE under reducing conditions (Fig. 2a). It is important to point out that, as described previously, PMM2-CDG patients had increased levels of hypoglycosylated forms of other hepatic proteins in plasma, such as antithrombin, $\alpha 1$-antitrypsin, FXI or transferrin [19], as verified in our patients by different methods, Western Blot, coagulometric assays, Q-TOF, or HPLC (Fig. 2b).

In order to verify that the aberrant FXII detected in PMM2-CDG is a hypoglycosylated form, plasma of PMM2-CDG and healthy subjects was treated with PNGase-F. This treatment eliminated the heterogeneity of FXII forms in plasma of PMM2-CDG patients, but the resulting FXII without N-glycans (aglycoFXII) was smaller than the aberrant FXII detected in plasma of patients (Fig. 2c). This result suggested that the hypoglycosylated FXII form detected in plasma of PMM2-CDG patients contains one N-glycan (1 N-glycan-FXII), which is consistent with the size of an $\mathrm{N}$-glycan $(2 \mathrm{kDa})$ and the presence of hypoglycosylated forms of other proteins (antithrombin, FXI, transferrin) in these patients $[21,22]$. Moreover, these results also 


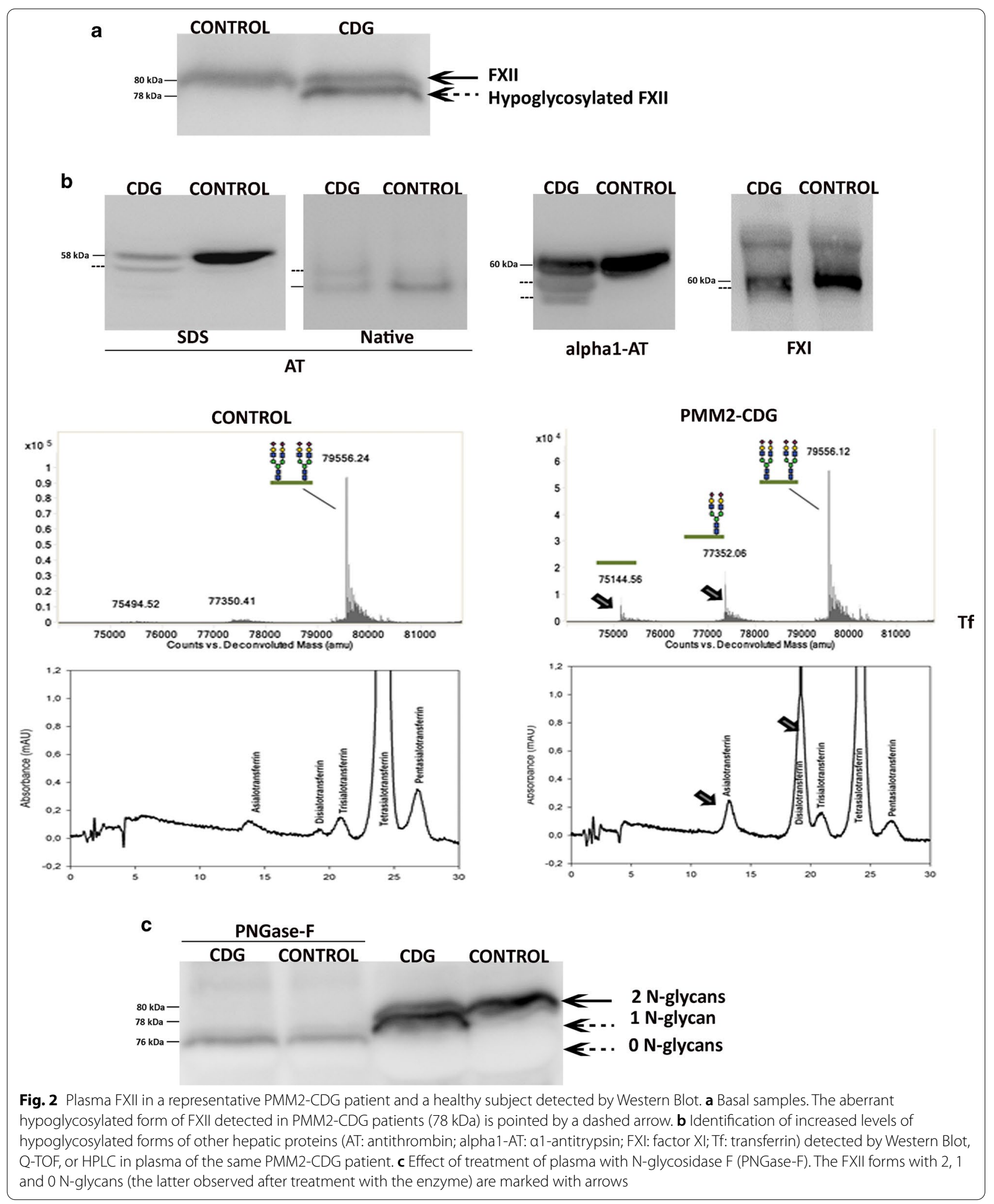


suggested that PMM2-CDG patients had no aglyco-FXII detectable by Western Blot in circulation (Fig. 2c).

The levels of the $1 \mathrm{~N}$-glycan-FXII form detected in PMM2-CDG patients were related to the glycosylation defect of each patient, accordingly to the direct correlation observed between the ratio between the two glycoforms determined densitometrically (1 N-glycan/2 N-glycan) and the levels of asialotransferrin $\left(\mathrm{r}^{2}=0.731\right.$ and $\left.p=0.003\right)$. Indeed, an indirect correlation was also observed between this ratio and the anticoagulant activity of antithrombin $\left(\mathrm{r}^{2}=0.456\right.$ and $\left.p=0.046\right)$. Thus, patients with higher asialotransferrin values had lower antithrombin anticoagulant function and high levels of the hypoglycosylated FXII (Fig. 3).

\section{Clinical characteristics of patients according to FXII}

Vascular events were reported in 20 out of these 46 PMM2-CDG patients. Most of these events were stroke-like $(n=12)$, but also thrombosis $(n=6)$ and hemorrhages $(n=3)$ were recorded. No relationship of these vascular events with the levels of FXII were observed (Table 2). However, the presence of vascular events significantly associated with higher levels of hypoglycosylated FXII forms, although this was also true for all markers of hypoglycosylation like asialotransferrin or disialotransferrin, antithrombin or FXI levels (Table 2).

Finally, no angioedema events were reported in our cohort of PMM2-CDG patients.

\section{Activation of FXII}

FXII activation in plasma of PMM2-CDG patients was studied by evaluating the presence of the FXIIa heavy chain by Western Blot, using different concentrations of two activators: silica and DXS. Three interesting results were observed:

Firstly, there was no basal activation of FXII in plasma samples of PMM2-CDG patients (Fig. 4).

Second, after FXII activation the heterogeneity of FXII forms observed in PMM2-CDG patients disappeared, and only a single FXIIa heavy chain was detected, which
Table 2 Levels of FXII and the hypoglycosylated form of FXII as well as other markers of hypoglycosylation in PMM2-CDG patients according to the presence or absence of vascular events

\begin{tabular}{llll}
\hline & $\begin{array}{l}\text { Vascular } \\
\text { events } \\
(\mathbf{N}=\mathbf{2 0})\end{array}$ & $\begin{array}{l}\text { No vascular } \\
\text { complications } \\
(\mathbf{N = 2 6 )}\end{array}$ & $\boldsymbol{p}$ \\
\hline FXII:C (\%) & $113 \pm 48 \%$ & $120 \pm 41 \%$ & 0.728 \\
1 N/2 N FXII ratio & $1.26 \pm 0.04$ & $0.54 \pm 0.28$ & 0.003 \\
Asialo-transferrin (\%) & $33.5 \pm 12.9$ & $21.9 \pm 15.1$ & 0.077 \\
Disialo-trasnferrin (\%) & $12.8 \pm 8.4$ & $4.5 \pm 5.3$ & 0.013 \\
Antithrombin (\%) & $40.6 \pm 23.9$ & $72.0 \pm 31.1$ & 0.001 \\
FXI (\%) & $35.6 \pm 26.0$ & $74.9 \pm 36.30$ & 0.001 \\
\hline
\end{tabular}

Statistically significant $p$-values $(\leq 0.05)$ are shown in italics

was similar to that found in healthy controls (Fig. 4). This result means that the hypoglycosylation of FXII in PMM2-CDG patients preferentially affected Asn414, which is located in the light chain. Therefore, most if not all aberrant FXII of PMM2-CDG patients was glycosylated at Asn230 (Fig. 4).

Third, dose-dependent studies using the two activators suggested that the hypoglycosylated form of FXII detected in PMM2-CDG patients was activated in a similar way to the 2-N-glycan form (Fig. 4).

\section{Recombinant FXII expression}

Analysis of the secreted protein in the culture medium of $\mathrm{S} 2$ cells transfected with the plasmids containing the human F12 cDNA was carried out by Western Blot. Cells transfected with the wild-type plasmid produced high levels of FXII in the culture medium. The secretion and electrophoretic mobility of the Lys 309 variant was similar to the wild-type (Fig. 5). In contrast, the expression of the variants affecting $\mathrm{N}$-glycosylation sequons was significantly reduced in the culture medium, especially for the mutant affecting the 230 sequon and the double mutant. Of note, the variant affecting the 414 sequon showed high secretion levels, although they did not reach

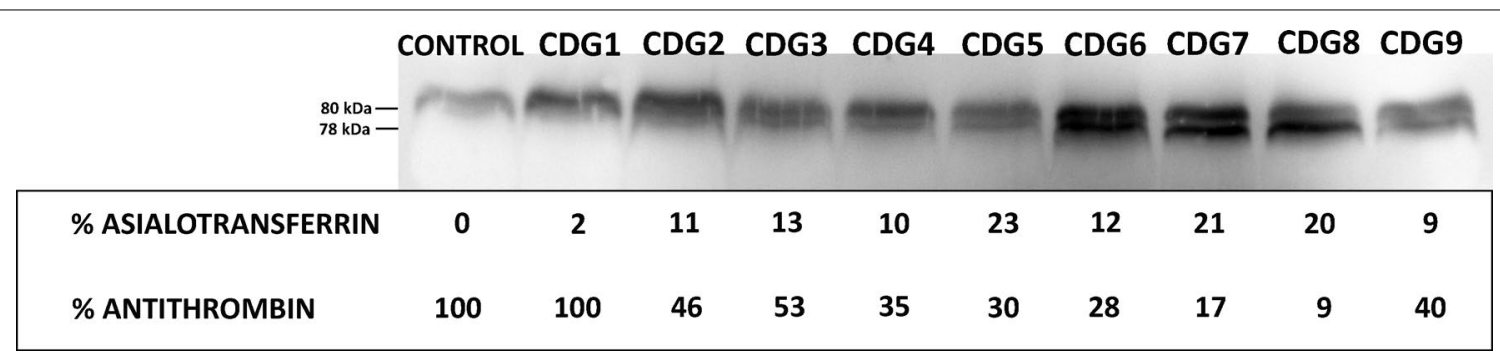

Fig. 3 Levels of hypoglycosylated FXII and its relationship with the levels of asialotransferrin (a-Tf) and antithrombin (AT). PMM2 variants in 9 PMM2-CDG patients and a healthy control. Haploins: haploinsuficiency 

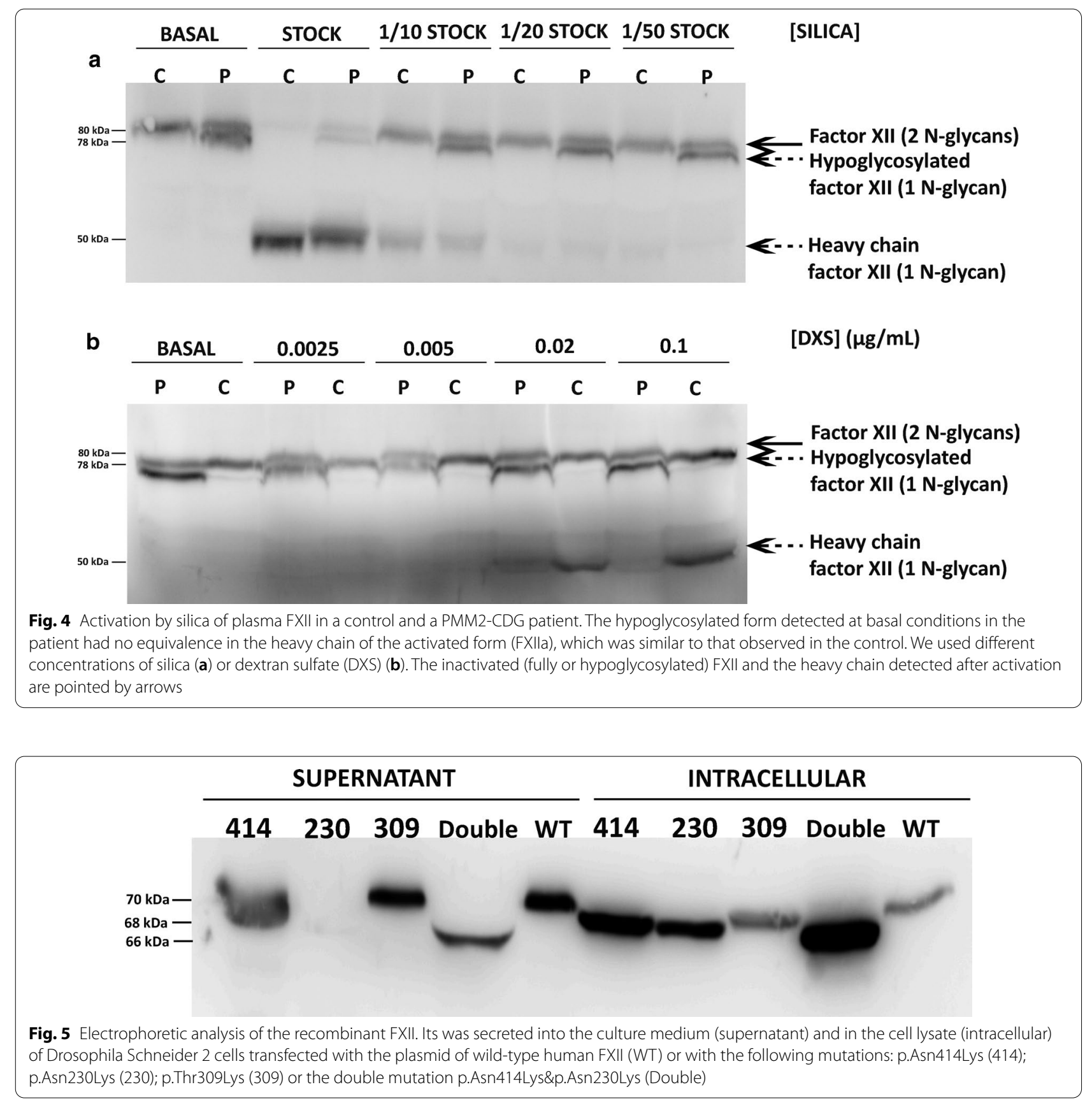

the levels observed in cells transfected with the wild-type or the Lys309 plasmids (Fig. 5). Furthermore, the variants with a single mutation affecting an $\mathrm{N}$-glycan sequon (230 and 414) showed greater electrophoretic mobility than the wild-type or the Lys309 forms, while the double mutant generated the recombinant FXII with greater electrophoretic mobility in SDS gels, consistent with the loss of one or two N-glycans (Fig. 5).

The study of the intracellular FXII forms of the S2 cells showed that mutants lacking any $\mathrm{N}$-glycosylation and therefore presenting hypoglycosylation (230, 414 and double mutant 230-414) had higher intracellular retention as compared to the wild-type or the p.Lys309 forms (Fig. 5). In addition, the size differences observed in the protein secreted into the medium were maintained in the intracellular protein, again being the double mutant, without any $\mathrm{N}$-glycosylation the form with faster mobility and increased intracellular retention (Fig. 5). 


\section{Discussion}

PMM2-CDG is a rare devastating disease affecting mainly children [25]. The defect in the generation of the $\mathrm{N}$-glycan precursor caused by the impaired activity of PMM2 alters the N-glycosylation of potentially all $\mathrm{N}$-glycoproteins. The key role of $\mathrm{N}$-glycosylation for many proteins explains why the resulting hypoglycosylation could affect either the levels or the function of multiple proteins from different organs and tissues [26]. Consequently, the resulting phenotype will have strong biological consequences, particularly during the embryonic development. That explains the severe clinical phenotype of PMM2-CDG patients. It can be associated with multivisceral involvement and also affects the hemostatic system. Thrombosis but also bleeding events, have been described in PMM2-CDG patients as soon as during the perinatal period [25-27]. Our objective was to study the hemostatic system in PMM2-CDG due to the key role that $\mathrm{N}$-glycosylation plays in many different plasma proteins from the hemostatic system [28]. Indeed, several coagulation factors are affected in PMM2-CDG, in particular antithrombin, FXI, protein $C$, protein $S$ and FIX [27]. In this study, we focused on FXII, an emerging hemostatic molecule that has been involved also in hereditary angioedema. Our data support that FXII levels does not associate to vascular events in these patients, which is in accordance to the low relevance that this factor has for physiological hemostasia both in human patients and animal models [29]. However, we observed an association between these complications and the levels of the hypoglycosylated form of FXII detected in PMM2CDG patients. This could be explained by the severity of the disease, as hypoglycosylated transferrin and low levels of antithrombin and FXI are also associated to vascular events in our cohort. However, a potential role of this aberrant FXII favouring these events might also be speculated. Indeed, our study aimed to better understand the $\mathrm{N}$-glycosylation of this molecule and its relevance for a correct folding and secretion to the plasma and its activation and role in haemostasis and thrombosis.

The identification of a hypoglycosylated form of FXII in plasma of PMM2-CDG patients, the association of the levels of this aberrant FXII with antithrombin and asialotransferrin levels, and the further analysis of FXII, by using a variety of methods (PNGAse-F treatment, activation by silica or DXS) resulted in the following observations:

First, aglyco-FXII was not detected in plasma of PMM2-CDG patients suggesting that the presence of $\mathrm{N}$-glycosylation is crucial for the folding and secretion of FXII.

Second, our results support that most if not all the hypoglycosylated form of FXII detected in plasma of
PMM2-CDG was glycosylated at Asn230, but lacks the $\mathrm{N}$-glycan at Asn414, although further studies including glycoproteomic analysis must be done to demonstrate the aberrant glycosylation of FXII in PMM2-CDG patients. This specific hypoglycosylation might be explained by two mechanisms. (1) The glycosylation of Asn230 is crucial for the correct folding and secretion of FXII, or (2) Asn230 is much more efficiently glycosylated under restrictive concentrations of the glycan precursor. Despite the expression in insect cells have some limitations, such as the different glycan composition than that of mammal cells, the results obtained in the recombinant model support the first hypothesis as the FXII variant altering residue 414 was more efficiently secreted than the 230 variant. Indeed, Asn230 is conserved in FXII across species (83\%) (Additional file 1: Fig. 1). Two species (Usus americanus and Mustela putorius furo) have no Asn at 230 position but instead of this glycosylation site they present an additional $\mathrm{N}$-sequon only 22 residues far from Asn230 (in Asn252), which is also located in the heavy chain (Additional file 1: Fig. 1).

Finally, our study also provides interesting information concerning hereditary angioedema, a disease where FXII plays a significant role. The defective O-glycosylation associated with the F12 p.Thr309Lys mutation has been involved in an increased sensitivity to the activation of FXII and the development of hereditary angioedema [17]. We might expect that the absence of a complete $\mathrm{N}$-glycan at the C-terminus of FXII might also have the same consequences. However, the data obtained from PMM2CDG patients do not support an increased activation of the aberrant hypoglycosylated FXII with low doses of the tested activators (silica or DXS) and importantly, no PMM2-CDG patient neither from our cohort nor from the literature has ever developed angioedema to our knowledge. These data support recent studies showing the key role of the $\mathrm{N}$-terminal domain of FXII in the activation of this molecule [30]. However, we must remember that most PMM2-CDG patients have not been exposed to the triggering factors that cause angioedema in carriers of the p.Thr309Lys mutation (pregnancy, oral contraceptives...) [31], and on the other hand that marked edemas were already reported in CDG patients [32-36].

To sum up, our study has revealed new information on FXII, one emerging molecule in different disorders, namely the critical role that glycosylation at Asn230 has in the efficient secretion of FXII to the plasma. Moreover, our study opens new perspectives on the relevance that glycosylation might have in the contact pathway. Finally, CDG patients undoubtedly are an excellent source of information to understand the relevance of $\mathrm{N}$-glycosylation for other glycoproteins and disorders. 
However, we also must take into account that, as any other complex disease, CDG may be accompanied by compensatory mechanisms that might affect $\mathrm{N}$-linked glycan synthesis or the functional consequences of one aberrant molecule.

\section{Conclusions}

In this study, we characterized the glycosylation of FXII, the first element of the contact pathway in PMM2CDG patients. These patients present high levels of a homogeneous FXII hypoglycosylated form without $\mathrm{N}$-glycosylation at Asn414. This, and the results obtained in the recombinant model support that $\mathrm{N}$-glycosylation at Asn230 is essencial for the correct folding and/or secretion of FXII. Finally, the results confirm that this glycoform is similarly activated than fully glycosylated FXII, explaining the absence of angioedema in PMM2CDG patients.

\section{Supplementary information}

Supplementary information accompanies this paper at https://doi. org/10.1186/s13023-020-01564-9.

Additional file 1: Fig. 1. Conservation of N-glycosylation sequons in FXII from 12 species.

\section{Abbreviations}

CDG: Congenital disorders of glycosylation; PPM2: Phosphomannomutase 2; FXIl: Factor XII; HAE: Hereditary angioedema; FXI: Factor XI; BK: Bradykinin; FXIla: Activated FXII; PTM: Post-translational modification; PAGE: Polyacrilamide electrophoresis; DTT: Dithiothreitol; PNGaseF: N-glycosidase F; DXS: Dextran sulfate; Asp: Asparagine; Lys: Lysine; Thr: Threonine; SFM: Serum-free Express Five.

\section{Acknowledgements}

We thank the technical assistance of Jose Padilla.

\section{Authors' contributions}

RL-G was responsible for the experimental work. AL-L colleted FXII-HAE patients' samples and revised the manuscript. MP assisted to the performance of the recombinant model. AM assisted with the technical work. MS and DB collected CDG patients' and clinical data and contributed with the revision. VR provided patients and critically reviwed the manuscrist. RL-G, MEM-B and $J C$ performed and designed the research, analyzed the data and wrote the paper. JE and $\mathrm{W}$ analyzed the data and contributed to critical revision of the manuscript. All authors read and approved the final manuscript.

\section{Funding}

MEMB has a postdoctoral fellowship of Universidad de Murcia, Spain. This study was supported by the Fundación Séneca (19873/GERM/15), CIBERER (ACCI18-04; ER19P5AC765/2019) and Sociedad Española de Trombosis y Hemostasia (SETH: grupos emergentes).

\section{Availability of data and materials}

Not applicable.

\section{Ethics approval and consent to participate}

This study is run in accordance with the recommendations from the Declaration of Helsinki and has received ethical, regulatory and institutional approvals at national, regional and site level for each participating country, as required. All participants or their legal representative provide informed consent before their enrolment in the registry.
Consent for publication

Not applicable.

\section{Competing interests}

The authors declare that they have no competing interests.

\section{Author details}

${ }^{1}$ Servicio de Hematología y Oncología Médica, Hospital Universitario Morales Meseguer, Centro Regional de Hemodonación, Universidad de Murcia, IMIB-Arrixaca, CIBERER, Ronda de Garay S/N, 30003 Murcia, Spain. ${ }^{2}$ Instituto de Investigación Sanitaria del Hospital La Paz (IdiPaz), Madrid, Spain. ${ }^{3}$ Centre for Biomedical Network Research On Rare Diseases (CIBERER) U-754, Hospital Universitario La Paz, Madrid, Spain. ${ }^{4}$ Centre for Biomolecular Sciences, School of Pharmacy, University of Nottingham, Nottingham NG7 2RD, England. ${ }^{5}$ Departamento de Neurología Pediátrica, Departamento de Bioquímica Clínica, Instituto de Investigación Pediátrica-Hospital Sant Joan de Déu, CIBERER U-703, Barcelona, Spain. ${ }^{6}$ Laboratoire D'Hématologie, AP-HP, Hôpital Necker-Enfants Malades, Paris, France. ${ }^{7}$ UMR-S1176, Université Paris-Saclay, INSERM, 94276 Le Kremlin-Bicêtre, France.

Received: 17 July 2020 Accepted: 25 September 2020

Published online: 09 October 2020

\section{References}

1. Pauer HU, Renné T, Hemmerlein B, Legler T, Fritzlar S, Adham I, et al. Targeted deletion of murine coagulation factor $X I I$ gene-a model for contact phase activation in vivo. Thromb Haemost. 2004;92(3):503-8.

2. Maruyama H, Brooks MB, Stablein A, Frye A. Factor XII deficiency is common in domestic cats and associated with two high frequency F12 mutations. Gene. 2019;706:6-12.

3. Key NS. Epidemiologic and clinical data linking factors XI and XII to thrombosis. Hematology. 2014;2014(1):66-70.

4. Kleinschnitz C, Stoll G, Bendszus M, Schuh K, Pauer H-U, Burfeind P, et al. Targeting coagulation factor XII provides protection from pathological thrombosis in cerebral ischemia without interfering with hemostasis. J Exp Med. 2006;203(3):513-8. https://doi.org/10.1084/jem.20052458.

5. Kuijpers MJE, Van Der Meijden PEJ, Feijge MAH, Mattheij NJA, May F, Govers-Riemslag J, et al. Factor XII regulates the pathological process of thrombus formation on ruptured plaques. Arterioscler Thromb Vasc Biol. 2014:34(8):1674-80

6. Müller F, Mutch NJ, Schenk WA, Smith SA, Esterl L, Spronk HM, et al. Platelet polyphosphates are proinflammatory and procoagulant mediators in vivo. Cell. 2009;139(6):1143-56.

7. Xu Y, Cai TQ, Castriota G, Zhou Y, Hoos L, Jochnowitz N, et al. Factor Xlla inhibition by Infestin-4: in vitro mode of action and in vivo antithrombotic benefit. Thromb Haemost. 2013;111:694-704.

8. Yau JW, Liao P, Fredenburgh JC, Stafford AR, Revenko AS, Monia $\mathrm{BP}$, et al. Selective depletion of factor XI or factor XII with antisense oligonucleotides attenuates catheter thrombosis in rabbits. Blood. 2014;123(13):2102-7.

9. Larsson M, Rayzman V, Nolte MW, Nickel KF, Björkqvist J, Jämsä A, et al. Cardiovascular disease: a factor Xlla inhibitory antibody provides thromboprotection in extracorporeal circulation without increasing bleeding risk. Sci Transl Med. 2014;6:222ra17.

10. Matafonov A, Leung PY, Gailani AE, Grach SL, Puy C, Cheng Q, et al. Factor XII inhibition reduces thrombus formation in a primate thrombosis model. Blood. 2014;123(11):1739-46.

11. Bork K, Wulff K, Meinke P, Wagner N, Hardt J, Witzke G. A novel mutation in the coagulation factor 12 gene in subjects with hereditary angioedema and normal C1-inhibitor. Clin Immunol. 2011;141(1):31-5.

12. Dewald G, Bork K. Missense mutations in the coagulation factor XII (Hageman factor) gene in hereditary angioedema with normal C1 inhibitor. Biochem Biophys Res Commun. 2006;343(4):1286-9.

13. Kiss N, Barabás E, Várnai K, Halász A, Varga LÁ, Prohászka Z, et al. Novel duplication in the F12 gene in a patient with recurrent angioedema. Clin Immunol. 2013;149(1):142-5. 
14. Gelincik A, Demir S, Olgaç M, Karaman V, Toksoy G, Çolakołlu B, et al. Idiopathic angioedema with F12 mutation: is it a new entity. Ann Allergy Asthma Immunol. 2015:114:154-6.

15. Amiral J, Seghatchian J. The contact system at the crossroads of various key patho-physiological functions: update on present understanding, laboratory exploration and future perspectives. Transfus Apher Sci. 2019;58:216-22.

16. Mitra N, Sinha S, Ramya TNC, Surolia A. N-linked oligosaccharides as outfitters for glycoprotein folding, form and function. Trends Biochem Sci. 2006;31:156-63.

17. Björkqvist J, de Maat S, Lewandrowski U, Di Gennaro A, Oschatz C, Schönig K, et al. Defective glycosylation of coagulation factor XII underlies hereditary angioedema type III. J Clin Investig. 2015;125(8):3132-46.

18. Ng BG, Freeze HH. Perspectives on glycosylation and its congenital disorders. Trends Genet. 2018;34:466-76.

19. Van Schaftingen $E$, Jaeken J. Phosphomannomutase deficiency is a cause of carbohydrate-deficient glycoprotein syndrome type I. FEBS Lett. 1995;377(3):318-20.

20. Babovic-Vuksanovic D, O'Brien JF. Laboratory diagnosis of congenital disorders of glycosylation type I by analysis of transferrin glycoforms. Mol Diagn Ther. 2007;11:303-11.

21. Pascreau T, Morena-Barrio ME, Lasne D, Serrano M, Bianchini E, Kossorotoff $M$, et al. Elevated thrombin generation in patients with congenital disorder of glycosylation and combined coagulation factor deficiencies. J Thromb Haemost. 2019;17:1798-807.

22. de la Morena-Barrio ME, Martínez-Martínez I, de Cos C, Wypasek E, Roldán $V$, Undas A, et al. Hypoglycosylation is a common finding in antithrombin deficiency in the absence of a SERPINC1 gene defect. J Thromb Haemost. 2016;14(8):1549-60.

23. Kanaji T, Okamura T, Osaki K, Kuroiwa M, Shimoda K, Hamasaki N, et al. A common genetic polymorphism (46 C to T substitution) in the 5'untranslated region of the coagulation factor XII gene is associated with low translation efficiency and decrease in plasma factor XII level. Blood. 1998;91(6):2010-4.

24. Schneider CA, Rasband WS, Eliceiri KW. NIH Image to ImageJ: 25 years of image analysis. Nat Methods. 2012;9:671-5.

25. Jaeken J. Congenital disorders of glycosylation. In: Blau N, Hoffmann GF, Leonard JV, Clarke JTR, editors. Physician's guide to the treatment and follow-up of metabolic diseases. New York: Springer; 2006. p. 217-20.

26. Stanley P, Taniguchi N, Aebi M. Chapter 9. N-glycans, essentials of glycobiology. In: Essentials of Glycobiology, 2nd edn; 2017.
27. Linssen M, Mohamed M Wevers RA, Lefeber DJ, Morava E Thrombotic complications in patients with PMM2-CDG. Mol Genet Metab. 2013:109(1):107-11.

28. Preston RJS, Rawley O, Gleeson EM, O'Donnell JS. Elucidating the role of carbohydrate determinants in regulating hemostasis: Insights and opportunities. Blood. 2013;121:3801-10.

29. Maas C, Renné T. Coagulation factor XII in thrombosis and inflammation. Blood. 2018. https://doi.org/10.1182/blood-2017-04-569111.

30. Ivanov I, Matafonov A, Sun MF, Mohammed BM, Cheng Q, Dickeson SK, et al. A mechanism for hereditary angioedema with normal C1 inhibitor: an inhibitory regulatory role for the factor XII heavy chain. Blood. 2019;133(10):1152-63.

31. Magerl M, Germenis AE, Maas C, Maurer M. Hereditary angioedema with normal C1 inhibitor: update on evaluation and treatment. Immunol Allergy Clin N Am. 2017;37:571-84.

32. Noelle V, Knuepfer M, Pulzer F, Schuster V, Siekmeyer W, Matthijs G, et al, Unusual presentation of congenital disorder of glycosylation type 1a: congenital persistent thrombocytopenia, hypertrophic cardiomyopathy and hydrops-like aspect due to marked peripheral oedema. Eur J Pediatr. 2005; 164(4):223-6

33. Panigrahy N, Lingappa L, Ramadevi AR, Venkatlakshmi A. Congenital disorder of glycosylation (CDG) presenting as non-immune hydrops fetalis. Indian J Pediatr. 2016;83:359-60.

34. Van De Kamp JM, Lefeber DJ, Ruijter GJG, Steggerda SJ, Den Hollander NS, Willems SM, et al. Congenital disorder of glycosylation type la presenting with hydrops fetalis. J Med Genet. 2007;44(4):277-80

35. Edwards M, McKenzie F, O'Callaghan S, Somerset D, Woodford P, Spilsbury $\mathrm{J}$, et al. Prenatal diagnosis of congenital disorder of glycosylation type la (CDG-la) by cordocentesis and transferrin isoelectric focussing of serum of a $27-$ week fetus with non-immune hydrops. Prenat Diagn. 2006:26(10):985-8.

36. De Koning TJ, Toet M, Dorland L, De Vries LS, Van Den Berg IET, Duran $M$, et al. Recurrent nonimmune hydrops fetalis associated with carbohydrate-deficient glycoprotein syndrome. J Inherit Metab Dis. 1998;21(6):681-2

\section{Publisher's Note}

Springer Nature remains neutral with regard to jurisdictional claims in published maps and institutional affiliations.
Ready to submit your research? Choose BMC and benefit from:

- fast, convenient online submission

- thorough peer review by experienced researchers in your field

- rapid publication on acceptance

- support for research data, including large and complex data types

- gold Open Access which fosters wider collaboration and increased citations

- maximum visibility for your research: over $100 \mathrm{M}$ website views per year

At BMC, research is always in progress.

Learn more biomedcentral.com/submissions 